\title{
Multicriteria games and potentials
}

\author{
Fioravante Patrone · Lucia Pusillo • Stef Tijs
}

Received: 13 December 2005 / Accepted: 9 January 2007 /

Published online: 12 April 2007

(C) Sociedad de Estadística e Investigación Operativa 2007

\begin{abstract}
In this note we study how far the theory of strategic games with potentials, as reported by Monderer and Shapley (Games Econ Behav 14:124-143, 1996), can be extended to strategic games with vector payoffs, as reported by Shapley (Nav Res Logist $Q$ 6:57-61, 1959). The problem of the existence of pure approximate Pareto equilibria for multicriteria potential games is also studied.
\end{abstract}

Keywords Noncooperative potential games $\cdot$ Multicriteria Pareto equilibria · Approximate Pareto equilibria $\cdot$ Supermodularity

Mathematics Subject Classification (2000) 91A10

\section{Introduction}

Monderer and Shapley (1996) introduced a subclass of strategic games for which a potential exists. Such potential games turn out to have interesting properties, among which the existence of pure (approximate) Nash equilibria in the case of upper

\footnotetext{
F. Patrone

DIPTEM, Sezione Metodi e Modelli Matematici, Facoltà di Ingegneria, University of Genoa,

P. le Kennedy, pad. D, 16129 Genoa, Italy

e-mail: patrone@diptem.unige.it

L. Pusillo $(\varangle) \cdot$ S. Tijs

DIMA, Department of Mathematics, University of Genoa, via Dodecaneso 35, 16146 Genoa, Italy

e-mail: pusillo@dima.unige.it

S. Tijs

CentER and Department of Econometrics and Operations Research, Tilburg University,

P.O. Box 90153, 5000 LE Tilburg, The Netherlands

e-mail: s.h.tijs@uvt.nl
} 
bounded potentials. Further, they show that there is an interesting relation between potential games and congestion games introduced by Rosenthal (1973). Our list of references shows that the Monderer-Shapley paper inspired many game theorists.

The purpose of this paper is to investigate the possibility of extending the potential theory to multicriteria strategic games introduced by Shapley (1959) and also studied by Borm et al. (1989). Since the extension of the theory of potential games to multicriteria situations is turning out to be obvious in the case we know the extension to the bicriteria situation, in this paper we restrict ourselves mostly to bicriteria situations.

The outline of the paper is as follows. In Sect. 2, we introduce bicriteria games with a potential. In Sect. 3 we show that in the case the strategy spaces are finite, such a game has at least one pure Pareto equilibrium. Further, for multicriteria games with an upper bounded (vector) potential, it turns out that $\epsilon$-Pareto equilibria in pure strategies exist. In Sect. 4, we give a characterization of zero-sum bicriteria games with a potential and discuss their relations with supermodular bicriteria games. Section 5 concludes with some suggestions for further research.

\section{Bicriteria games and potentials}

A strategic bicriteria game is a tuple $\Gamma=\left\langle N,\left(A_{i}\right)_{i \in N},\left(u_{i}\right)_{i \in N}\right\rangle$, where $N$ is the set of players, $A_{i}$ is the strategy space for player $i \in N, A$ is the Cartesian product $\prod_{k \in N} A_{k}$ of the strategy spaces $\left(A_{i}\right)_{i \in N}$, and $u_{i}: A \rightarrow \mathbb{R}^{2}$ is the utility function for player $i$.

We call $\Gamma=\left\langle N,\left(A_{i}\right)_{i \in N},\left(u_{i}\right)_{i \in N}\right\rangle$ a potential game if there exists a map $P: A \rightarrow \mathbb{R}^{2}$ such that, for all $i \in N, a_{i}, b_{i} \in A_{i}$, and $a_{-i} \in A_{-i}:=\prod_{j \in N \backslash\{i\}} A_{j}$, we have

$$
u_{i}\left(a_{i}, a_{-i}\right)-u_{i}\left(b_{i}, a_{-i}\right)=P\left(a_{i}, a_{-i}\right)-P\left(b_{i}, a_{-i}\right) .
$$

In the classical theory, potential games can be also characterized with the aid of conditions on paths of strategy profiles (cf. Monderer and Shapley 1996 and Voorneveld 1999). Since the extension to bicriteria games is straightforward, we do not pay attention to this.

Special classes of potential games are bicriteria coordination games and bicriteria dummy games. A game $\left\langle N,\left(A_{i}\right)_{i \in N},\left(u_{i}\right)_{i \in N}\right\rangle$ is a coordination game if the utility functions of all players are the same. Clearly, for such a game, there is a potential $P: A \rightarrow \mathbb{R}^{2}$. Take, e.g., $P=u_{1}$.

The game $\left\langle N,\left(A_{i}\right)_{i \in N},\left(u_{i}\right)_{i \in N}\right\rangle$ is a dummy game if $u_{i}\left(a_{i}, a_{-i}\right)=u_{i}\left(b_{i}, a_{-i}\right)$ for all $i \in N, a_{i}, b_{i} \in A_{i}$, and $a_{-i} \in A_{-i}$, that is, the strategy choice of player $i$ does not influence his payoff. A potential for such a game is the null function $P$ with $P(a)=(0,0)$ for each $a \in A$.

Note that $\left\langle N,\left(A_{i}\right)_{i \in N},\left(u_{i}+u_{i}^{\prime}\right)_{i \in N}\right\rangle$ is a bicriteria potential game with potential $P+P^{\prime}$ if $\left\langle N,\left(A_{i}\right)_{i \in N},\left(u_{i}\right)_{i \in N}\right\rangle$ is a bicriteria potential game with potential $P$ and $\left\langle N,\left(A_{i}\right)_{i \in N},\left(u_{i}^{\prime}\right)_{i \in N}\right\rangle$ a bicriteria potential game with potential $P^{\prime}$.

In particular, the sum of a bicriteria coordination game and a bicriteria dummy game is a potential bicriteria game.

The converse also holds as we see in the next theorem. This theorem extends the result of Facchini et al. (1997) and Slade (1994). 
Theorem 2.1 (Decomposition theorem). Let $\left\langle N,\left(A_{i}\right)_{i \in N},\left(u_{i}\right)_{i \in N}\right\rangle$ be a bicriteria potential game. Then there is a coordination game $\left\langle N,\left(A_{i}\right)_{i \in N},(P)_{i \in N}\right\rangle$ and a dummy game $\left\langle N,\left(A_{i}\right)_{i \in N},\left(D_{i}\right)_{i \in N}\right\rangle$ such that $u_{i}=P+D_{i}$ for each $i \in N$.

Proof Take for $P$ a potential of the original game and define $D_{i}=u_{i}-P$. Then from (2.1) it follows that $\left\langle N,\left(A_{i}\right)_{i \in N},\left(D_{i}\right)_{i \in N}\right\rangle$ is a dummy game.

Example 2.2 Consider the game where $N$ consists of two players (the row player and column player) and each player has two strategies (player I: row 1 (A) and row 2 (B); player II: column 1 (C) and column 2 (D)), and where the utility functions are described in the following payoff matrix:

\begin{tabular}{l|c|c|}
\multicolumn{2}{c}{$\mathrm{C}$} & \multicolumn{2}{c}{$\mathrm{D}$} \\
\cline { 3 - 4 } A & $(0,3)(0,5)$ & $(4,5)(1,3)$ \\
\cline { 2 - 3 } B & $(0,0)(1,3)$ & $(5,5)(3,4)$ \\
\cline { 2 - 3 }
\end{tabular}

A decomposition of the game into a coordination game and a dummy game is given by

\begin{tabular}{|l|l|}
\hline$(0,3)(0,5)$ & $(4,5)(1,3)$ \\
\hline$(0,0)(1,3)$ & $(5,5)(3,4)$ \\
\hline
\end{tabular}

\begin{tabular}{|l|l|}
\hline$(0,3)(0,3)$ & $(1,1)(1,1)$ \\
\hline$(0,0)(0,0)$ & $(2,1)(2,1)$ \\
\hline
\end{tabular}$+\quad$\begin{tabular}{|l|l|}
\hline$(0,0)(0,2)$ & $(3,4)(0,2)$ \\
\hline$(0,0)(1,3)$ & $(3,4)(1,3)$ \\
\hline
\end{tabular}

Bicriteria coordination game Bicriteria dummy game

\section{Existence of (approximate) pure Pareto equilibria}

We recall the definition of Pareto equilibrium of a bicriteria game.

Definition 3.1 Let $\Gamma=\left\langle N,\left(A_{i}\right)_{i \in N},\left(u_{i}\right)_{i \in N}\right\rangle$ be a bicriteria strategic game. Then $\hat{a} \in \prod_{i \in N} A_{i}$ is called a Pareto equilibrium ( $P E$ for short) of the game if, for each $i \in N$, we have $\hat{a}_{i} \in P B\left(\hat{a}_{-i}\right)$. Here the set $P B\left(\hat{a}_{-i}\right)$ of Pareto best answers to $\hat{a}_{-i}$ is the set of $a_{i} \in A_{i}$ such that $u_{i}\left(b_{i}, \hat{a}_{-i}\right) \notin u_{i}\left(a_{i}, \hat{a}_{-i}\right)+\left(\mathbb{R}_{+}^{2} \backslash\{0\}\right)$ for all $b_{i} \in A_{i}$.

We denote by $P E(\Gamma)$ the set of Pareto equilibria of $\Gamma$.

The following theorem, which extends a result of Monderer and Shapley (1996), says that finite bicriteria potential games have at least one Pareto equilibrium.

Theorem 3.2 Let $\Gamma=\left\langle N,\left(A_{i}\right)_{i \in N},\left(u_{i}\right)_{i \in N}\right\rangle$ be a finite bicriteria potential game. Then there exists an $\hat{a} \in \prod_{i \in N} A_{i}$ such that $\hat{a}$ is a pure Pareto equilibrium of the game $\Gamma$.

Proof Let $P: A \rightarrow \mathbb{R}^{2}$ be a potential for $\Gamma$. 
Take $\hat{a} \in \operatorname{argmax}_{a \in A}\left(P_{1}(a)+P_{2}(a)\right)$. Next, we prove that $\hat{a} \in P E(\Gamma)$. Suppose that $\hat{a} \notin P E(\Gamma)$. Then there are $i \in N$ and $a_{i} \in A_{i}$ such that

$$
u_{i}\left(a_{i}, \hat{a}_{-i}\right)-u_{i}(\hat{a}) \in \mathbb{R}_{+}^{2} \backslash\{0\} .
$$

But then

$$
P\left(a_{i}, \hat{a}_{-i}\right)-P(\hat{a}) \in \mathbb{R}_{+}^{2} \backslash\{0\},
$$

so

$$
\sum_{k=1}^{2}\left(P_{k}\left(a_{i}, \hat{a}_{-i}\right)-P_{k}(\hat{a})\right)>0,
$$

which is in contradiction with the definition of $\hat{a}$.

The proof of the following theorem is left to the reader.

Theorem 3.3 Let $\Gamma=\left\langle N,\left(A_{i}\right)_{i \in N},\left(u_{i}\right)_{i \in N}\right\rangle$ be a bicriteria strategic potential game with potential function $P$. Then

$$
P E(\Gamma)=P E\left(\Gamma^{P}\right)
$$

where $\Gamma^{P}=\left\langle N,\left(A_{i}\right)_{i \in N},(P)_{i \in N}\right\rangle$.

Now we concentrate ourselves on the existence of approximate Pareto equilibria for bicriteria potential games. We introduce the following definition for approximate equilibria.

Definition 3.4 Let $\Gamma=\left\langle N,\left(A_{i}\right)_{i \in N},\left(u_{i}\right)_{i \in N}\right\rangle$ be a bicriteria strategic game and $\epsilon>0$. Then $\hat{a} \in \prod_{i \in N} A_{i}$ is called an $\epsilon$-Pareto equilibrium of the game $\Gamma$ if, for each $i \in N$, we have $\hat{a}_{i} \in P B^{\epsilon}\left(\hat{a}_{-i}\right)$. Here the set $P B^{\epsilon}\left(\hat{a}_{-i}\right)$ of $\epsilon$-Pareto best answers to $\hat{a}_{-i}$ is the set of $a_{i} \in A_{i}$ such that $u_{i}\left(b_{i}, \hat{a}_{-i}\right) \notin u_{i}\left(a_{i}, \hat{a}_{-i}\right)+\mathbb{R}_{+, \epsilon}^{2}$ for all $b_{i} \in A_{i}$.

Here $\mathbb{R}_{+, \epsilon}^{2}=\mathbb{R}_{+}^{2} \backslash([0, \epsilon] \times[0, \epsilon])$.

We further denote by $P E^{\epsilon}(\Gamma)$ the set of $\epsilon$-Pareto equilibria of a game $\Gamma$.

Definition 3.5 For $f: X \rightarrow \mathbb{R}$, we define

$$
\operatorname{argsup}_{x \in X}^{\epsilon} f(x)=\left\{y \in X: f(y) \geq \sup _{x \in X} f(x)-\epsilon\right\} .
$$

The following theorem extends a result of Norde and Tijs (1998).

Theorem 3.6 Let $\Gamma=\left\langle N,\left(A_{i}\right)_{i \in N},\left(u_{i}\right)_{i \in N}\right\rangle$ be a bicriteria strategic potential game. Suppose that the potential functions are upper bounded. Then $P E^{\epsilon}(\Gamma) \neq \emptyset$ for all $\epsilon>0$. 
Proof Let $P$ be a potential for $\Gamma$ and $\epsilon>0$.

Take $\hat{a} \in \operatorname{argsup}_{a \in A}^{\epsilon}\left(P_{1}(a)+P_{2}(a)\right)$. We prove that $\hat{a} \in P E^{\epsilon}(\Gamma)$.

Take $i \in N$ and $a_{i} \in A_{i}$. Suppose that

$$
u_{i}\left(a_{i}, \hat{a}_{-i}\right) \in u_{i}(\hat{a})+\mathbb{R}_{+, \epsilon}^{2} .
$$

Then

$$
P\left(a_{i}, \hat{a}_{-i}\right)-P(\hat{a})=u_{i}\left(a_{i}, \hat{a}_{-i}\right)-u_{i}(\hat{a}) \in \mathbb{R}_{+, \epsilon}^{2} .
$$

But then

$$
\sum_{k=1}^{2}\left(P_{k}\left(a_{i}, \hat{a}_{-i}\right)-P_{k}(\hat{a})\right)>\epsilon,
$$

a contradiction to the definition of $\hat{a}$. So, for each $i \in N, \hat{a}_{i} \in P B^{\epsilon}\left(\hat{a}_{-i}\right)$, i.e., $\hat{a} \in P E^{\epsilon}(\Gamma)$.

Example 3.7 Let $\left\langle\{1,2\},(0,1),(0,1), u_{1}, u_{2}\right\rangle$ be the 2-person potential game on the open unit square with $u_{1}\left(x_{1}, x_{2}\right)=\left(x_{1}, x_{1}^{2}\right)$ and $u_{2}\left(x_{1}, x_{2}\right)=\left(x_{2}, x_{2}^{3}\right)$.

This game has a potential $P:(0,1) \times(0,1) \rightarrow \mathbb{R}^{2}$ given by $P\left(x_{1}, x_{2}\right)=\left(x_{1}+x_{2}\right.$, $\left.x_{1}^{2}+x_{2}^{3}\right)$ for all $\left(x_{1}, x_{2}\right) \in(0,1) \times(0,1)$. This game has no Pareto equilibria but there exist $\epsilon$-Pareto equilibria for every $\epsilon>0$.

We shall say that a game is weakly determined if the set of its $\epsilon$-Pareto equilibria is nonempty for each $\epsilon>0$. The next theorem implies that $\Gamma$ is (weakly) determined if and only if $\Gamma^{P}$ is (weakly) determined.

Theorem 3.8 Let $\Gamma=\left\langle N,\left(A_{i}\right)_{i \in N},\left(u_{i}\right)_{i \in N}\right\rangle$ be a bicriteria strategic potential game with potential $P$ and $\Gamma^{P}=\left\langle N,\left(A_{i}\right)_{i \in N},(P)_{i \in N}\right\rangle$. Then $P E^{\epsilon}(\Gamma)=P E^{\epsilon}\left(\Gamma^{P}\right)$ for all $\epsilon \geq 0$.

Proof Let $D_{i}=u_{i}-P$ for each $i \in N$.

The following five assertions are equivalent:

(i) $\hat{a} \in P E^{\epsilon}(\Gamma)$;

(ii) $u_{i}\left(a_{i}, \hat{a}_{-i}\right) \notin u_{i}(\hat{a})+\mathbb{R}_{+, \epsilon}^{2}$ for all $i \in N$ and $a_{i} \in A_{i}$;

(iii) $P\left(a_{i}, \hat{a}_{-i}\right)+D_{i}\left(a_{i}, \hat{a}_{-i}\right) \notin P(\hat{a})+D_{i}(\hat{a})+\mathbb{R}_{+, \epsilon}^{2}$ for all $i \in N$ and $a_{i} \in A_{i}$;

(iv) $P\left(a_{i}, \hat{a}_{-i}\right) \notin P(\hat{a})+\mathbb{R}_{+, \epsilon}^{2}$ for all $i \in N$ and $a_{i} \in A_{i}$;

(v) $\hat{a} \in P E^{\epsilon}\left(\Gamma^{P}\right)$.

Monderer and Shapley (1996) relate potential games with congestion models introduced by Rosenthal (1973). We can generalize them to the multicriteria case in a straightforward way.

For the classical finite potential games and congestion games, it was shown (Monderer and Shapley 1996; Voorneveld 1999) that every finite potential game is isomorphic to a congestion game. One can extend this result to bicriteria situations by adapting the beautiful proof of Voorneveld and using Theorem 2.1. 
Monderer and Shapley (1996) present a class of Cournot games which consists of potential games. It is also possible to connect multicriteria Cournot games of special type with multicriteria potential games in a straightforward way.

\section{Zero-sum bicriteria games and supermodularity}

A bicriteria game $\Gamma=\left\langle\{1,2\}, A_{1}, A_{2}, u_{1}, u_{2}\right\rangle$ with $u_{1}(a)+u_{2}(a)=(0,0)$ for each $a \in A_{1} \times A_{2}$ is called a zero-sum bicriteria game.

For a zero-sum bicriteria game with an exact potential, the payoff functions have a special form as we see in the next theorem. It extends a result of Potters et al. (1999).

Theorem 4.1 Let $\Gamma=\left\langle\{1,2\}, A_{1}, A_{2}, u_{1}, u_{2}\right\rangle$ be a two-person zero-sum bicriteria game. Then the following assertions are equivalent:

(i) $\Gamma$ is a potential game;

(ii) there exist maps $f: A_{1} \rightarrow \mathbb{R}^{2}$ and $g: A_{2} \rightarrow \mathbb{R}^{2}$ such that $u_{1}\left(a_{1}, a_{2}\right)=f\left(a_{1}\right)-$ $g\left(a_{2}\right)=-u_{2}\left(a_{1}, a_{2}\right)$ for all $\left(a_{1}, a_{2}\right) \in A_{1} \times A_{2}$ (Separation property).

Proof To see that (ii) implies (i), it suffices to note that $P: A \rightarrow \mathbb{R}^{2}$ given by $P\left(a_{1}, a_{2}\right)=f\left(a_{1}\right)+g\left(a_{2}\right)$ for each $\left(a_{1}, a_{2}\right) \in A_{1} \times A_{2}$ is a potential for the game $\Gamma$.

Conversely, suppose that (i) holds and let $P: A_{1} \times A_{2} \rightarrow \mathbb{R}^{2}$ be a potential. Take $\left(a_{1}^{*}, a_{2}^{*}\right) \in A_{1} \times A_{2}$ and define $f: A_{1} \rightarrow \mathbb{R}^{2}$ and $g: A_{2} \rightarrow \mathbb{R}^{2}$ by

$$
\begin{array}{ll}
f\left(a_{1}\right)=1 / 2\left(P\left(a_{1}, a_{2}^{*}\right)-u_{2}\left(a_{1}, a_{2}^{*}\right)\right) & \text { for each } a_{1} \in A_{1} \\
g\left(a_{2}\right)=1 / 2\left(P\left(a_{1}^{*}, a_{2}\right)-u_{1}\left(a_{1}^{*}, a_{2}\right)\right) & \text { for each } a_{2} \in A_{2} .
\end{array}
$$

Since $P$ is a potential, we have that, for each $\left(a_{1}, a_{2}\right) \in A_{1} \times A_{2}$,

$$
u_{1}\left(a_{1}, a_{2}\right)-u_{1}\left(a_{1}^{*}, a_{2}\right)=P\left(a_{1}, a_{2}\right)-P\left(a_{1}^{*}, a_{2}\right)
$$

and

$$
u_{2}\left(a_{1}, a_{2}\right)-u_{2}\left(a_{1}, a_{2}^{*}\right)=P\left(a_{1}, a_{2}\right)-P\left(a_{1}, a_{2}^{*}\right),
$$

from which follows that

$$
u_{1}\left(a_{1}, a_{2}\right)=P\left(a_{1}, a_{2}\right)-2 g\left(a_{2}\right), \quad u_{2}\left(a_{1}, a_{2}\right)=P\left(a_{1}, a_{2}\right)-2 f\left(a_{1}\right) .
$$

From these last two equalities and from the fact that $u_{2}=-u_{1}$ we obtain

$$
P\left(a_{1}, a_{2}\right)=f\left(a_{1}\right)+g\left(a_{2}\right) \quad \text { for all }\left(a_{1}, a_{2}\right) \in A_{1} \times A_{2}
$$

and then

$$
u_{1}\left(a_{1}, a_{2}\right)=f\left(a_{1}\right)-g\left(a_{2}\right) \quad \text { for all }\left(a_{1}, a_{2}\right) \in A_{1} \times A_{2} .
$$

Hence, (ii) holds.

Note that Theorem 4.1 extends the results for uni-criteria games in Theorem 1 of Branzei et al. (2003). In that paper, this separation theorem is used to show that each 
classical two-person zero-sum game with an exact potential is strategically equivalent to a supermodular game.

For more details about supermodularity, see Topkis (1998). This result can be extended for special bicriteria zero-sum potential games, namely, for bicriteria games with parallel criteria. We say that $\Gamma=\left\langle\{1,2\}, A_{1}, A_{2}, u_{1},-u_{1}\right\rangle$ has parallel criteria if, for all $a_{1}, a_{1}^{\prime} \in A_{1}$ and $a_{2}, a_{2}^{\prime} \in A_{2}$, we have:

(i) $u_{11}\left(a_{1}, a_{2}\right) \geq u_{11}\left(a_{1}^{\prime}, a_{2}\right) \Leftrightarrow u_{12}\left(a_{1}, a_{2}\right) \geq u_{12}\left(a_{1}^{\prime}, a_{2}\right)$;

(ii) $u_{11}\left(a_{1}, a_{2}\right) \geq u_{11}\left(a_{1}, a_{2}^{\prime}\right) \Leftrightarrow u_{12}\left(a_{1}, a_{2}\right) \geq u_{12}\left(a_{1}, a_{2}^{\prime}\right)$.

Here $u_{1}=\left(u_{11}, u_{12}\right)$.

By adapting the proof of Branzei et al., we straightforwardly obtain the following:

Theorem 4.2 Each zero-sum bicriteria potential game with parallel criteria is strategically equivalent to a supermodular game.

\section{Concluding remarks}

In this paper, we define and study bicriteria strategic games with a potential. It turns out that, under certain conditions, these games have pure approximate Pareto equilibria. Moreover, there is a close relation between these games and bicriteria congestion games.

For zero-sum bicriteria games, the separation property holds. A subclass of these games can be related to supermodular games.

Monderer and Shapley (1996) also consider finite ordinal potential games which turn out to have pure Nash equilibria. Extensions to infinite games are studied in Voorneveld and Norde (1997) and in Norde and Patrone (2002). What about an extension to multicriteria ordinal potential games and to other classes of potential games?

Let us call a bicriteria game $G=\left(X_{1}, X_{2}, u_{1}, u_{2}\right)$ an ordinal potential bicriteria game if there exists a function $P: X_{1} \times X_{2} \rightarrow \mathbb{R}^{2}$ such that

$$
u_{1}\left(x_{1}, x_{2}\right)-u_{1}\left(y_{1}, x_{2}\right) \geq 0 \Leftrightarrow P\left(x_{1}, x_{2}\right)-P\left(y_{1}, x_{2}\right) \geq 0
$$

and

$$
u_{2}\left(x_{1}, x_{2}\right)-u_{2}\left(x_{1}, y_{2}\right) \geq 0 \Leftrightarrow P\left(x_{1}, x_{2}\right)-P\left(x_{1}, y_{2}\right) \geq 0
$$

for all

$$
x_{1}, y_{1} \in X_{1}, x_{2}, y_{2} \in X_{2} .
$$

Then one can prove (similarly to Theorem 3.2) that every finite ordinal potential game possesses a Pareto equilibrium.

There are still many questions left that can be interesting topics for possible further research:

(i) In Peleg et al. (1996), an axiomatic approach is taken for the set of Nash equilibria corresponding to the points where the potential is maximal.

An axiomatization of the Pareto optimum set corresponding to a potential for a multicriteria game could be a very interesting question. 
(ii) Extensions of well-posedness results in Margiocco and Pusillo (2007) to multicriteria potential games may be possible and interesting.

(iii) The work of Facchini et al. (1997) can be extended to Bayesian multicriteria potential games.

(iv) The work of Voorneveld et al. (1998) on sequential production situations and potentials is possibly extendable to bicriteria situations.

Acknowledgements This paper is partially supported by MIUR (Ministero dell'Istruzione, dell'Università e della Ricerca) and by GNAMPA (Gruppo Nazionale per l'Analisi Matematica, la Probabilità e Applicazioni).

The authors gratefully thank three anonymous referees and Rodica Branzei for their helpful comments and suggestions to improve the paper.

\section{References}

Branzei R, Mallozzi L, Tijs S (2003) Supermodular games and potential games. J Math Econ 39:39-49

Borm PEM, Tijs SH, van den Aarssen JCM (1989) Pareto equilibria in multiobjective games. Methods Oper Res 60:303-312

Facchini G, Van Megen F, Borm P, Tijs S (1997) Congestion models and weighted Bayesian potential games. Theory Decis 42:193-206

Margiocco M, Pusillo L (2007) Stackelberg well-posedness and hierarchical potential games. In: Jorgensen S, Quincampoix M, Vincent TL (eds) Advances in dynamic game theory. Numerical methods, algorithms and applications to ecology and economics. Annals of the international society of dynamic games, vol 9 (to appear)

Monderer D, Shapley LS (1996) Potential games. Games Econ Behav 14:124-143

Norde H, Patrone F (2002) A potential approach for ordinal games. Top 9:69-75

Norde H, Tijs S (1998) Determinateness of strategic games with potential. Math Methods Oper Res 48:377-385

Peleg B, Potters J, Tijs S (1996) Minimality of consistent solutions for strategic games, in particular for potential games. Econ Theory 7:81-93

Potters J, Raghavan TES, Tijs S (1999) Pure equilibrium strategies for stochastic games via potential functions. Report No. 9910, Department of Mathematics, University of Nijmegen, The Netherlands

Rosenthal RW (1973) A class of games possessing pure strategy Nash equilibria. Int J Game Theory 2:65-67

Shapley LS (1959) Equilibrium points in games with vector payoffs. Nav Res Logist Q 6:57-61

Slade ME (1994) What does an oligopoly maximize?. J Ind Econ 42:45-61

Topkis D (1998) Supermodularity and complementarity. Princeton University Press, Princeton

Voorneveld M (1999) Potential games and interactive decisions with multiple criteria. Dissertation series No. 61, CentER of Economic Research, Tilburg University, The Netherlands

Voorneveld M, Norde H (1997) A characterization of ordinal potential games. Games Econ Behav 19:235242

Voorneveld M, Tijs S, Mallozzi L (1998) Sequential production situations and potentials In: GarciaJurado I, Patrone F, Tijs S (eds) Game practice: contributions from applied game theory. Kluwer Academic, Dordrecht 